\title{
Information Fusion for Estimation of Summer MIZ Ice Concentration from SAR Imagery
}

\author{
Donna Haverkamp, Member, IEEE, and Costas Tsatsoulis, Senior Member, IEEE
}

\begin{abstract}
In this paper we define the concept of information fusion and show how we used it to estimate summer sea ice concentration in the marginal ice zone (MIZ) from single-channel SAR satellite imagery. We used data about melt stage, wind speed, and surface temperature to generate temporally-accumulated information, and fused this information with the SAR image, resulting in an interpretation of summer MIZ imagery. We also used the results of previous classifications of the same area to guide and correct future interpretations, thus fusing historical information with imagery and nonimagery data. We chose to study the summer MIZ since summer melt conditions cause classification based upon backscatter intensity to fail, as the backscatter of open water, thin ice, first-year ice, and multiyear ice overlap to a large degree. This makes it necessary to fuse various information and data to achieve proper segmentation and automated classification of the image. Our results were evaluated qualitatively and showed that our approach produces very good ice concentration estimates in the summer MIZ.
\end{abstract}

\section{INTRODUCTION}

$\mathbf{T}$ HE marginal ice zone (MIZ) is the dynamic edge of the ice pack, where one may observe a mix of water, ice floes (both first-year ice and multiyear ice, which is ice that has survived at least one melt season), and "background matrix" or "substrate," which consists of broken-up pieces of floes of sub-resolution size. This is a significant region of the oceans. Open water in the arctic regions generates heat flux as much as two orders of magnitude greater than that through multiyear ice [1], [2], making the detection of open water extremely important in terms of global climate.

The classification of SAR imagery of the summer MIZ cannot be performed using backscatter information alone, since, depending upon a number of factors, the scene may exhibit different backscatter tendencies in the resident classes, resulting in differing image characteristics. The backscatter contrast between first-year and multiyear ice is mostly stable only from October through May; the summer season is characterized by nearly indistinguishable backscatter for all ice types. Surface melt affects the backscatter of the ice adversely, and different ice thickness cannot be distinguished based upon

Manuscript received June 15, 1998; revised November 20, 1998. This work was supported in part by a NASA Graduate Student Fellowship in Global Change Research NGT-30189, and in part by Naval Research Laboratory Contract N00014-95-C-6038.

D. Haverkamp was with the Department of Electrical Engineering and Computer Science, The University of Kansas, Lawrence, KS 66045-2228 USA. She is now with Information Systems Division, Harris Corporation, Melbourne, FL 32902.

C. Tsatsoulis is with the Department of Electrical Engineering and Computer Science, The University of Kansas, Lawrence, KS 66045-2228 USA (e-mail: tsatsoul@eecs.ukans.edu).

Publisher Item Identifier S 0196-2892(99)03450-6. backscatter alone. Once the melt season begins, the signatures of the different ice types quickly begin to move closer together. After converging, they remain indistinguishable until midsummer. Melt and drain cycles cause multiple backscatter reversals in the response of first-year and multiyear ice until the end of the summer, when the backscatter once again stabilize [3]-[6]. Consequently, neither the dynamic ranges produced in imaging these scenes nor the dynamic ranges of the resident classes of these scenes are consistent. The segmentation and classification of scenes imaged during this timespan is extremely difficult. Any strict classification based upon hardcoded expected intensities will fail. Even if a proper segmentation of the image is achieved, class labeling becomes a problem because the labels cannot be assigned based upon the image intensity alone. The highest-intensity portion of the image is not always the same class; no intensity-grouped portion of the image is consistently representative of the same class over the course of a summer season. An excellent intensity-based segmentation of the image is definitely a good starting point for image classification, but intensity alone cannot serve as the basis of a fully automated classification.

However, ancillary data and information sources can provide a context for the interpretation of a given scene and allow automated classification. Consequently, we turn to data fusion approaches. Data fusion can occur at three different levels [7]: data level fusion is performed at the single-element level (e.g., pixel) - two or more corresponding images may be added, subtracted, or somehow combined using more complex numeric methods; feature level fusion is performed using symbolic representations of the different data sources or images, typically using artificial intelligence techniques; finally, decision level fusion combines different theories or evidence generated from the different data sources to generate some conclusion about the scene.

A number of data fusion techniques have been utilized for image classification. These have been mostly concerned with achieving a one-to-one correspondence of different sensor channels or even different sensor types and using patterns across these different bands to characterize the classes within the image (which is why it is often referred to as multisensor or multisource fusion). Either many of these approaches require the training of a neural network to recognize the patterns of different classes, or they utilize some other supervised means, via the use of either exemplary class data or human participation. The imagery type we were working with (ERS1 images) is single-channel and traditional multisource fusion techniques could not be applied. Additionally, because of the 
backscatter behavior of summer MIZ sea ice, images of the same area are inconsistent with respect to intensity not only in the long term (the relative backscatter of the different classes varies over the course of the summer season) but also in the short term (the dynamic ranges of the classes in these scenes can vary from image to image).

In our work, we used a new kind of data of data fusion, information level fusion. Different pieces of information that are pertinent to the classification of the data are applied at appropriate times of the classification process. The image data are fused with different, heterogeneous data sources during the various classification stages; the result of a stage establishes which classification step will be applied next and which data source will be fused with the data. Temperature is a data source that we use to generate a piece of information-namely, the melt stage occurring in the imaged scene. Wind is a data source that is combined with the melt stage in order to determine the relative backscatter of the different classes within the image. This information is used to guide the segmentation and classification of the corresponding image. From the classified image, we generate data concerning floe sizes and ice and water percentages. We compare these to prior floe size distribution data and historical information concerning expected ice percentages for the corresponding week and location of the image under inspection. If the current classification is correct, we can record the current floe size distribution and ice coverage data for use in the next classification of the same area.

The approach to data fusion used here differs from traditional approaches in that instead of performing a "flat" data fusion in which Data A is used to generate Evidence A and Data B is used to generate Evidence B and then Evidence A and $\mathrm{B}$ are combined to make conclusions, it utilizes a hybrid of decision-, feature-, and data-level fusion techniques. The fusion is performed such that any piece of information is integrated into the classification at the appropriate time. The idea is that for data fusion to be effective, the arrangement of the solution must fit the characteristics of the available data. In the case of a difficult problem such as the classification of unstructured data like remotely sensed sea ice imagery, alternative sources of data or information must be studied to see how and in what format they can best be used to constrain the classification possibilities, and at what stage of the classification their utilization is appropriate.

Through detailed investigations of SAR imagery and literature and through discussion with experts in the field of SAR sea ice interpretation, data and information sources pertinent to the automated classification of summer MIZ imagery were identified. These sources are wind speed, temperature, prior ice floe statistics, and historically accumulated ice and water concentrations. Our method fuses these different information sources to achieve automated image classification. Surface temperatures are analyzed over time to deduce melt stage which, in combination with wind speed, can guide the segmentation and classification of an image into three classes: ice floes, background matrix (essentially ice), and water. The intermediate results are floe statistics and ice and water concentrations. These intermediate results are compared to prior floe statistics of the same area and historical ice and water concentrations to detect discrepancies or possible classification errors. If the classification is accepted as correct, a database is updated with the current classification results (both ice and water concentrations and floe size distributions). If not, the user is notified and requested to provide a correct classification of the area. Either way, final ice and water concentrations and floe size distributions are obtained and saved to assist in successive classifications of the same area.

Results show that an information fusion approach to image classification can assist greatly in the automation of the classification. Using other information pertinent to the scene, in addition to the image intensity itself, conclusions (or expectations) can be made about dominant image characteristics, which can in turn be exploited in order to segment and classify an image correctly, without the need for human intervention. An implementation of this approach applied to SAR imagery produced automated interpretation and generation of sea ice concentration statistics for summer MIZ data.

\section{BACKGROUND AND RELATED WORK}

\section{A. Data Fusion for Image Classification}

Data fusion combines different sources of raw data into a single set of information that is of greater benefit than the sum of its component parts [7]. Most data fusion techniques for image processing have been applied on multispectral data to combine multiple bands of concurrent imagery in order to classify a scene. A good number of these techniques are based upon neural network approaches. For example, one method used a back-propagation neural network to classify Landsat imagery using bands 2,4 , and 7 , and concatenating the data from all three bands at each pixel location [8]. In another system a back-propagation network was used to classify Landsat Thematic Mapper pixels, then a maximumlikelihood method was used to determine which classification among those generated by three different bands was correct [9]. See also [10] for an overview of many multisource data analysis techniques.

Other ways of fusing multispectral take advantage of correlation between spectral bands. One approach worked with polarimetric SAR data, which consists of $\mathrm{P}, \mathrm{L}$, and $\mathrm{C}$ bands in both copolarized and cross-polarized forms, and performed a multidimensional fuzzy clustering of the logarithm of the parameters composing the polarimetric covariance matrix of the multispectral data to segment the data into classes [11].

One unique approach was used on Thematic Mapper imagery and attempted to utilize combinations of bands that provide the best separability among classes. At each iteration, a band selection process was performed and one or two cover types were classified using that band or bands. Ancillary information, such as elevation, was also used for band selection and classification [12]. Another technique took into account knowledge about sensors, the multiple viewing notion, and the uncertainty and imprecision of models and data modeled with the possibility theory. A blackboard structure was used to allow "scene specialist" programs to control the processing of 
the image, and "conflict specialist" programs to resolve classification conflicts using spatial contextual knowledge of objects that involved multispectral data and intelligent analysis [13].

A data fusion technique for the classification of sea ice images used SAR data to generate estimates of the multiyear ice concentration, and applied this concentration as a seed value to the NASA TEAM algorithm that classifies sea ice using SSM/I images. The SAR data provided good multiyear ice estimates while the SSM/I data was used to compute first-year ice and open water estimates [14].

\section{B. Summer and MIZ Ice Backscatter Characteristics}

The summer ice season consists of several stages [4], beginning with the early melt stage in which the snow pack begins to undergo transformation due to melt/freeze cycling. It ends when moisture is continuously present in the snow cover. This also marks the beginning of the melt onset stage, which is characterized by dampness at the snow-ice interface and an average surface temperature near the melting point. This stage ends when most of the snow cover has become completely saturated, signaling the beginning of the advanced melt stage. From this point, the snow melts rapidly, ponding occurs, and drainage networks are created. The ice continues to decay until the freeze-up season begins.

A number of notable conclusions are made in [15] concerning the backscatter changes in the summer ice cover:

1) when the snow begins to melt in early summer, the contrast between multiyear and first-year signatures vanishes;

2) when no snow remains in the first-year ice, the winter contrast between first-year and multiyear ice is reversed, which causes the first-year ice returns to exceed the multiyear ice returns by a few decibels-the shift can occur in less than a week;

3) the melting of superimposed ice can cause the reversed contrast to disappear in less than a week;

4) as melt ponds increase in number, the multiyear ice return is again higher than the first-year ice return;

5) because of the rapid fluctuations in the backscatter of first-year and multiyear ice, it is very difficult to classify $\mathrm{C}$ - and $\mathrm{X}$-band radar images using intensitybased algorithms.

\section{Methodology}

Information fusion combines imagery of a scene with information about the image, the sensor, and the environment, to allow segmentation and classification. It requires a collection of heterogeneous data sources from which information can be generated using a variety of algorithms. Each piece of available information is incorporated into the image analysis system, as it becomes applicable. Some pieces are incorporated before the actual processing of an image even begins. Other pieces are generated at the beginning of processing in order to guide the entire classification and are incorporated at every step of the automatic classification procedure. Yet other pieces are incorporated after the classification has been completed, in order to evaluate the accuracy of the classification.

\begin{tabular}{ll}
\hline $72-141$ & Indexing Latitude/Longitude \\
1.02221 .52241 .02261 .0228 & Temperature Record \\
ADVANCED & Melt Stage \\
5.0229 & Wind Specd and Recording Date \\
226 & Date of Last Classification \\
PERC OW 7.75 ICE 92.25 & Last Classification Percentages \\
FLOE 5.00 5.00 8.00 9.0010.0015.00 & Corresponding Floe Distributions \\
\hline
\end{tabular}

Fig. 1. Ice database excerpt.

We selected the information and data sources following discussion with geophysicists at the National Ice Center, the Jet Propulsion Laboratory, and NASA Goddard Space Flight Center, and also based upon results described in the literature [4], [6], [13], [16]-[18]. The information needed is: state of melt, wind speed, acceptable ice concentration ranges, acceptable floe distribution ranges, and recent floe distributions. This information can be extracted from the following data sources: temperature records, wind speed records, daily ice charts, and a database of previous classifications. In the following, we discuss the information extraction and fusion steps.

\section{A. Information Extraction}

Temperature records and wind speed data provide much of the information that allows the automatic interpretation of summer MIZ SAR imagery. For our implementation, we used $2 \mathrm{~m}$ surface winds and $2 \mathrm{~m}$ air temperature data. Measurements are taken every $12 \mathrm{~h}$ for both wind and temperature, at approximately $100-\mathrm{km}$ resolution. Surface winds are modelgenerated through surface pressure, which is sampled by buoys in the Arctic Buoy Program. Temperatures are also sampled by the Arctic Buoy Program. Currently the accuracy of these values in unclear, especially due to the interpolation performed to estimate them over the whole Arctic.

By monitoring and analyzing the temperature in a given area over time, we can estimate the stage of melt in that area. Combined with wind speed, this information supplies us with the relative expected backscatter of ice floes, background matrix, and water. Because temperature and wind data are used for melt stage interpretation, a database containing updated wind and temperature information was maintained for continuous use over the course of the summer season. In addition, we developed a process by which the melt stage and wind can be used to predict the relative backscatter of the ice floes, background matrix, and water.

1) Ice Database: For any given latitude and longitude, we analyzed temperature records from the area to estimate the current melt state, and we utilized records of the most recently measured floe distributions in order to guide the classification of the area. To store these types of information, a database was maintained in which blocks of data were indexed to the nearest whole latitude and longitude point. Each block begins with a temperature record for the area and stores other information such as the state of melt (FREEZE, EARLY, ONSET, ADVANCED), the last recorded wind speed and the day on which that wind speed was recorded, the date of the last classification of the area, and the results of that last classification: the ice and water percentages and the floe size distributions. An excerpt is shown in Fig. 1. 


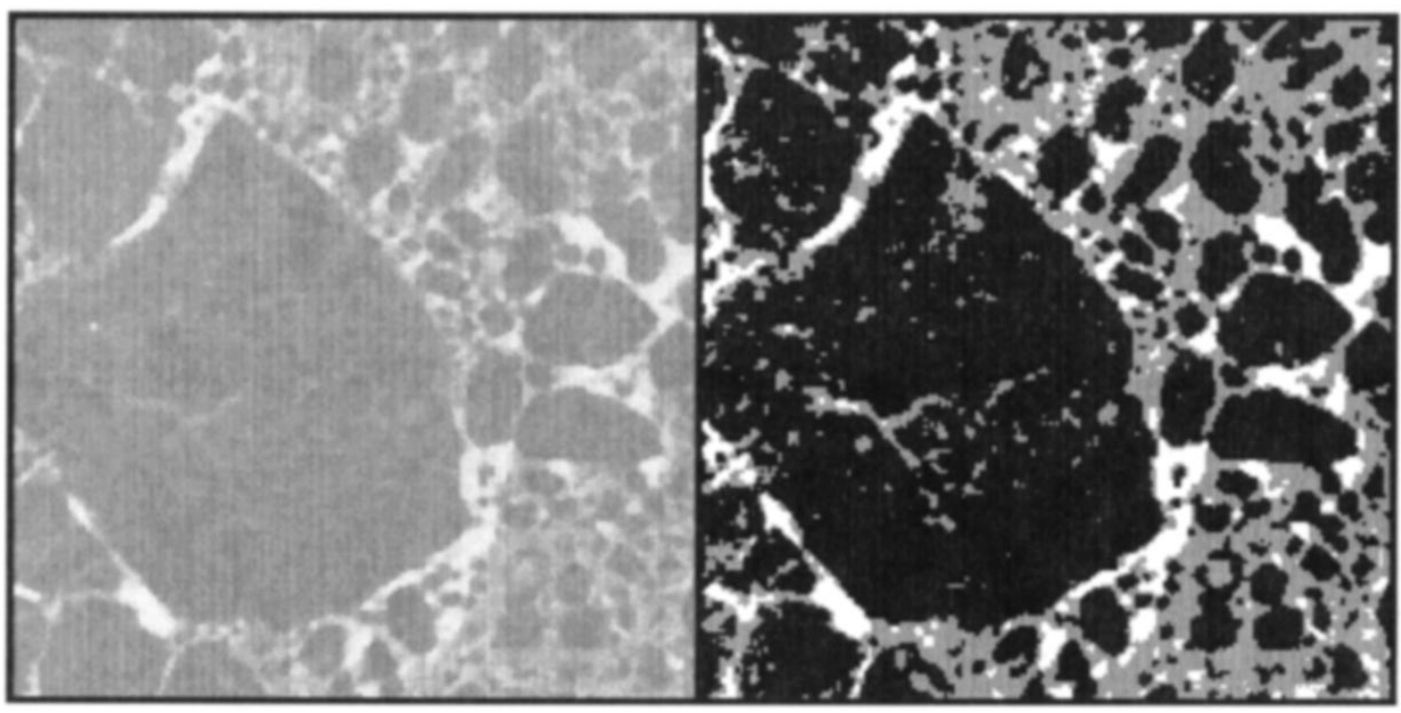

(a)

(b)

Fig. 2. (a) Original ERS-1 image segment (C) ESA 1991); (b) result of the three-level local dynamic thresholding.

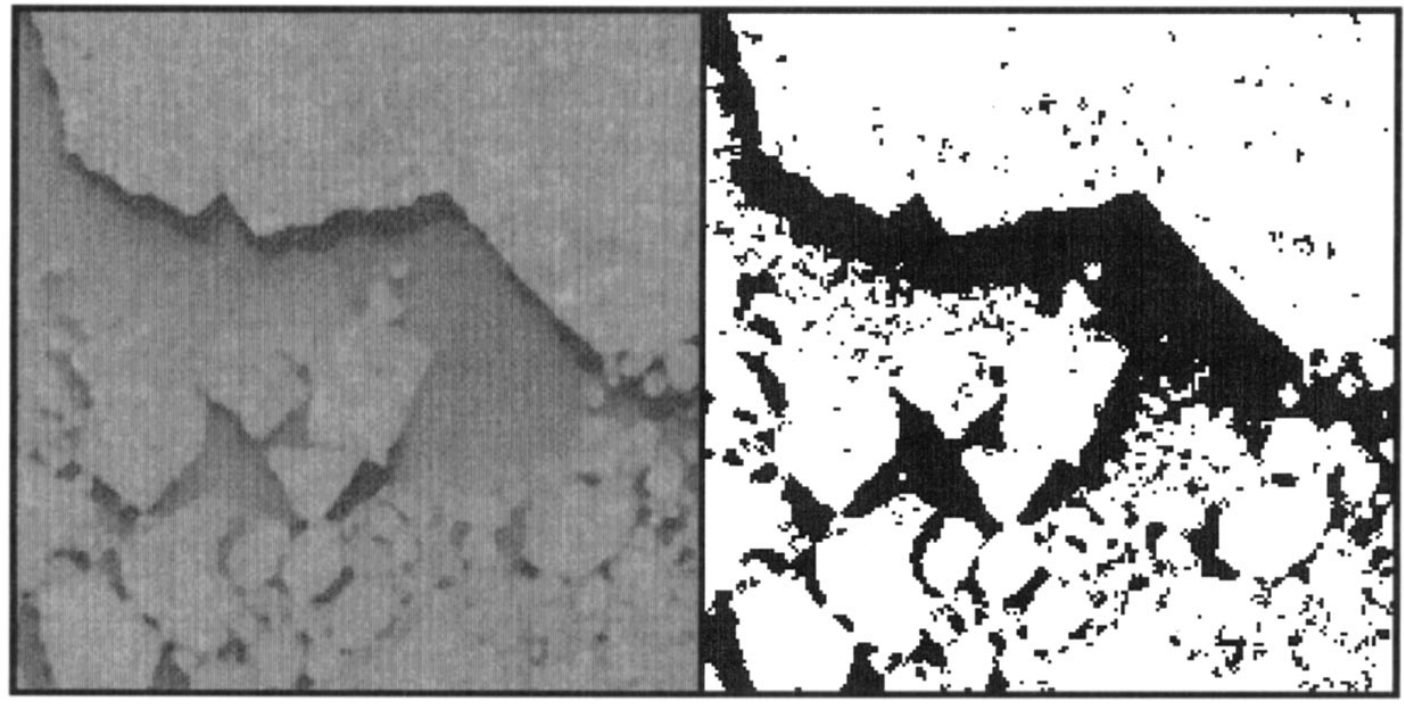

(a)

(b)

Fig. 3. Example of neighbor segmentation algorithm. (a) Original ERS-1 image segment (C) ESA 1991); (b) neighbor-segmented output. Although the water is both dark and bright, the neighbor segmentation manages to segment much of the bright water into the same class as the dark water.

The transitions between the different stages of melt are characterized through analyses of local temperature records. Given a temperature record of readings taken on a series of days prior to and including (or at least recent with respect to) the date of the scene being classified, we plot the ten last readings, perform a least-squares fit on the temperatures, and analyze the trend as rising, falling, fluctuating, or steady. We also compute the mean temperature and variance of the temperature data set.

Transition to Early Melt: After the winter freeze, the next stage must be early melt. If the average of the temperatures is very near to $0^{\circ} \mathrm{C}$, the temperatures do not exhibit a decreasing pattern, and the variance in the temperatures is quite small, then the area has entered the first stage of melt, or early melt. The transition is modeled as temperatures near to $0^{\circ} \mathrm{C}$ with some fluctuation (measured as the variance), but not an extensive amount of variation. We reject this evidence of early melt in the event that there is a decreasing trend present in the temperatures.

Transition to Melt Onset: After early melt, the next possible stage of melt is melt onset. This rule states that if the average of the temperature record is very near to $0^{\circ} \mathrm{C}$, and there is either an increasing trend in the temperatures or a very small variance in the temperatures and no decreasing trend, then the area has reached the melt onset stage. The transition is characterized by surface temperatures very near to the melting point and either an increasing trend in the record or a very low variance in the temperatures, indicating temperatures hovering near the melting point.

Transition to Advanced Melt: The next stage of melt is advanced melt. If the temperature is consistently above $0^{\circ} \mathrm{C}$, as indicated by an average temperature of greater than $0^{\circ}$ and 


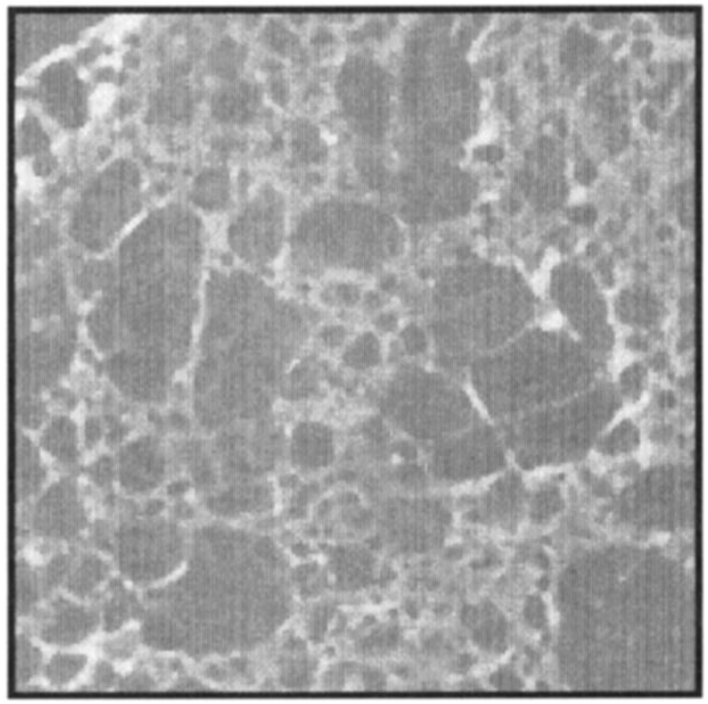

(a)

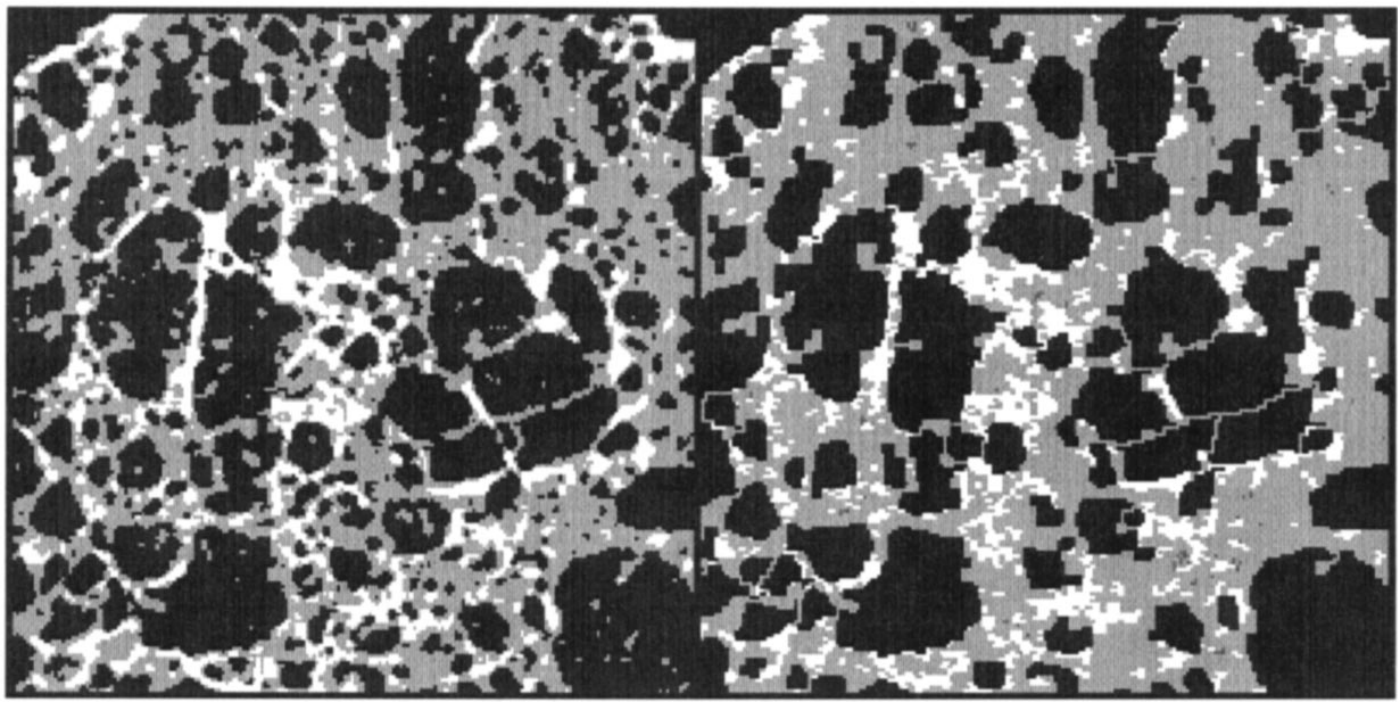

(b)

(c)

Fig. 4. (a) Original ERS-1 image segment (@ ESA 1991); (b) three-level segmented; (c) extracted floes after refinement.

either an increasing or steady temperature trend or no trend in the temperatures whatsoever but all temperatures in the record being greater than $0^{\circ}$, then the current state of melt can be safely assumed to be advanced melt.

Transition to Freeze-Up: The next transition is to winter freeze-up. This rule indicates that if the average temperature is somewhat below the freezing point and the temperatures indicate either a steady or decreasing trend or the average temperature is well below the freezing point, then the area has entered freeze-up. The transition is characterized by all temperatures being below $0^{\circ} \mathrm{C}$, relatively either far below zero or somewhat below zero and on a nonincreasing trend.

Each time the database is updated with a new temperature reading for some latitude and longitude, the meltstage information is re-evaluated to keep it current with the temperature record.

2) Parameterization: Depending upon the stage of melt being experienced by a given area, SAR imaging will result in different backscatter ranges for the different classes in the image (ice floes, background matrix, and water). In early melt, the floes have not yet been significantly affected by melt, so the relative backscatter of the resident classes is the same as for freeze-up: the floes are brighter than the background matrix, and the water is either the darkest (when the wind is low) or the brightest (when the wind is high). After melt onset occurs, the floes begin to be affected adversely by melt and the backscatters of the floes and the matrix begin to move closer together. The floes are now not strongly brighter than the matrix and, therefore, the effect of water within the matrix begins to be seen. When the water is bright (high wind), the mix-in of water with the matrix causes the matrix to become brighter than the floes. Alternatively, when the water is dark (low wind), the mix-in of the dark water with the matrix allows the matrix to remain darker than the floes. Later in the season, advanced melt is reached, and now the floes are consistently darker than the matrix. This leads to problems, however, when 
the water is dark (low wind). When this occurs, the floes are dark, the matrix is dark (due to the additional mix-in of the dark water), and the water is dark. For the purposes of image processing, the matrix and the floes are virtually indistinguishable. Either due to the sensor characteristics, or perhaps as another effect of advanced melt, sub-pixel size ice floes within the water areas sometimes strongly reflect the radar signal producing a backscatter which is more within the range of the ice floes and background matrix.

Parameters can be generated to guide the automated classification procedure. According to the melt stage and wind, the assumed relative backscatter of the resident classes of the image are used to guide a three-level segmentation, floe extraction, and classification of the image. Further investigations revealed that during advanced melt when the wind is low, the floes and matrix mix in such a way that they cannot be segmented apart. Additionally, in some of these scenes, even parts of the water areas mix in with the general backscatter range of the ice, leading to water areas which may be characterized by gray levels which are numerically distant from each other. In this case, a two-level segmentation using a neighborhood-based algorithm is preferred, providing a subsequent ice/no-ice classification without floe extraction.

\section{B. Image Segmentation}

Any image classification must have at its most basic level some means of dividing the image into its component classes. To accommodate all the different intensity ranges possible, a local thresholding technique was developed which splits an image into three unlabeled classes. This segmentation does not depend upon any predetermined threshold values or upon any parameters created in the parameterization process (derived from melt stage and wind). It simply assumes that there are three classes of some sort present in the image and attempts to segment the image accordingly, using a scheme based upon Gaussian curve-fitting of the histograms of smaller pieces of the image. This segmentation process attempts to define the three basic classes expected in summer, marginal ice zone imagery: ice floes, matrix, and water.

As indicated earlier, only segmentation between ice (ice floes and background matrix) and open water is possible under certain conditions. In this case, another segmentation procedure is selected as a result of the parameterization step. Because the water in these types of images often exhibits two very different general backscatters, it was determined that a neighbor-based algorithm would perform better than the dynamic thresholding procedure. The procedure developed is a hybrid neighbor- and gray-level-based segmentation in which gray values that neighbor each other extensively are grouped together, then those groups are grouped together using the same technique to obtain subgroups. A split point is then selected to divide the subgroups into two groups. A subsequent thresholding provides the two classes, ice and open water.

1) Dynamic Local Thresholding: The process used to perform a dynamic local thresholding of the image is based upon that used in [19], which divided an image into two basic regions, considered to be object and background. It
TABLE I

Floe Size Categories. "A” Is the Area of the Floe

\begin{tabular}{c|c|c|c}
\hline Very small & $\mathrm{A} \leq 100$ pixels & $\mathrm{A} \leq 1-\mathrm{km}^{2}$ & Bin 0 \\
\hline Small & $\mathrm{A} \leq 500$ pixels & $\mathrm{A} \leq 5-\mathrm{km}^{2}$ & $\mathrm{Bin} 1$ \\
\hline Medium & $\mathrm{A} \leq 5000$ pixels & $\mathrm{A} \leq 50-\mathrm{km}^{2}$ & Bin 2 \\
\hline Large & $\mathrm{A} \leq 12000$ pixels & $\mathrm{A} \leq 120-\mathrm{km}^{2}$ & Bin 3 \\
\hline Very large & $\mathrm{A} \leq 30000$ pixels & $\mathrm{A} \leq 300-\mathrm{km}^{2}$ & Bin 4 \\
\hline Vast & $\mathrm{A}>30000$ pixels & $\mathrm{A}>300 \mathrm{~km}^{2}$ & $\operatorname{Bin} 5$ \\
\hline
\end{tabular}

TABLE II

image Classification Evaluations. Out of 64 Points Possible for the 16 Images, 54 Were Awarded. The Average Score for the Images in the Data Set Was 3.375

\begin{tabular}{c|c|c|c}
\hline Image ID Number & Judge 1 Scorc & Judge 2 Scorc & Average Score \\
\hline 25025 & 2.5 & 3.5 & 3 \\
\hline 25026 & 4 & 4 & 4 \\
\hline 25027 & 4 & 4 & 4 \\
\hline 25028 & 3.5 & 4 & 3.75 \\
\hline 25053 & 2 & 3.5 & 2.75 \\
\hline 25054 & 2.5 & 3 & 2.75 \\
\hline 25055 & 2 & 3 & 2.5 \\
\hline 25056 & 2 & 3 & 2.5 \\
\hline 25658 & 3 & 3 & 3 \\
\hline 25659 & 3.5 & 3.5 & 3.5 \\
\hline 25660 & 2.5 & 3.5 & 3 \\
\hline 25661 & 3.5 & 4 & 3.75 \\
\hline 25662 & 3.5 & 3.5 & 3.5 \\
\hline 25362 & 4 & 4 & 4 \\
\hline 25363 & 4 & 4 & 4 \\
\hline 25364 & 4 & 4 & 3.375 \\
\hline Total Avg. Score: & $54 / 64$ possible & Avg. Image Score: & \\
\hline
\end{tabular}

has been adapted to segment the image into three classes. The version used in this work is an extension of that of [20] and the reader is referred to this work for details of the methodology. The image is first subdivided into many smaller regions; by using subregions of the image in the thresholding process, we can adapt to local variation within the image and preserve smaller-scale detail. A first pass through these regions is made to detect possibly bimodal histograms and approximate bimodal Gaussian curves for such regions. Criteria are applied to select those regions whose histograms are substantially bimodal. A bimodal Gaussian curve is approximated to represent the region. A second pass through the regions detects possibly trimodal histograms and approximates trimodal Gaussian curves for those regions in a similar manner. The bimodal and trimodal Gaussian curves approximated for any region are then tested for fit, and the best-fitting curve is used to characterize the region. Bimodal curves are used to calculate a single threshold using the Maximum Likelihood method, while trimodal curves are used to calculate two threshold values. The calculated thresholds are clustered into two groups-one to represent the division between Class 1 and Class 2, the other to represent the division between Class 2 and Class 3 . We then interpolate from regions with thresholds to other regions in the image to ensure that each region has two thresholds. A final interpolation is performed from the region down to the pixel level to provide each pixel with two threshold values. These interpolations assure smooth threshold transitions across the image. Finally, the actual thresholding is performed upon every pixel in the image, using the two threshold values determined for each 


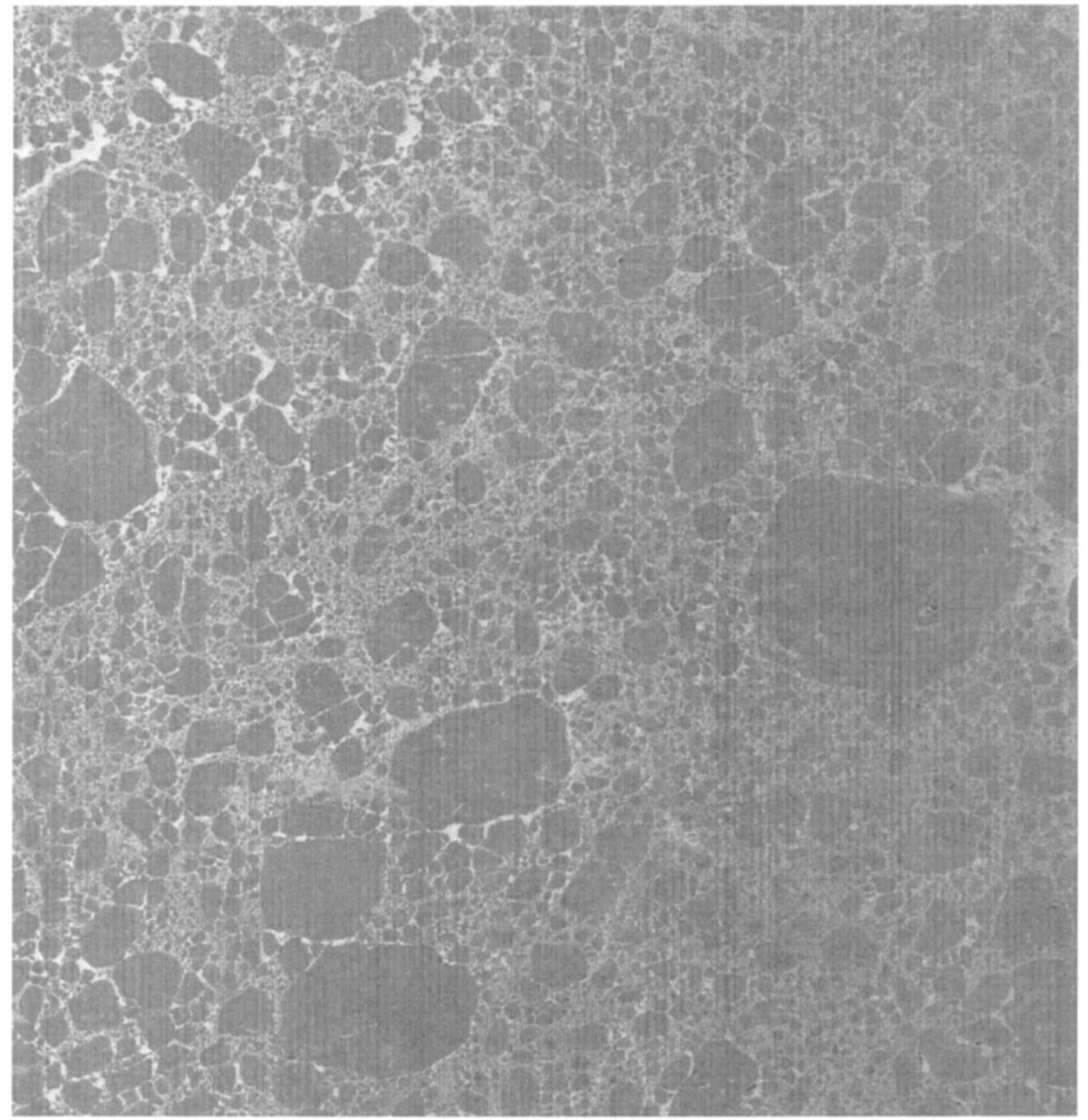

Fig. 5. Original ERS-1 image, median filtered, identification number 25026 (@ ESA 1991).

pixel. Each individual pixel is assigned to one of three classes. An example of how this algorithm segments an image can be seen in Fig. 2: Fig. 2(a) is the original image, while Fig. 2(b) is the segmented image. Note the preservation of detail.

2) Maximum-Neighbor Segmentation: This algorithm divides an image into two classes by utilizing a combination of co-occurrence matrix and nearest-neighbor techniques. Specifically, it adds up the number of times a pixel of gray level $y$ neighbors a pixel of gray level $x$ in any 8neighborhood direction. It then selects the maximum neighbor for each gray level (possibilities are 0-255). Each gray level is grouped with its maximum neighbor to form groupings of gray levels. Another grouping is then performed using maximum neighboring groups to form subgroupings. After this final subgrouping, a maximal split point is selected among the groups to maximize both the compactness and the diversity of each of the two groups found.
This method works independently of the dynamic range and, to some extent, uses the spatial proximity of a subclass within the image instead of merely gray level proximity. An example of the results attained by this algorithm can be seen in Fig. 3 . Fig. 3(a) is the original image, containing a water area which exhibits two very different backscatter ranges; Fig. 3(b) is the segmented image, which shows the two different backscatter subclasses combined as one class.

\section{Floe Refinement}

Floe distributions are used as an information source which indicates whether a current classification is valid: if the current floe size measurements are not consistent with the past floe size measurements of the same area (as stored in the ice database), it can indicate an error in the classification. For this reason, it is important that floe shapes be properly identified and isolated within the image. The parameters generated by 


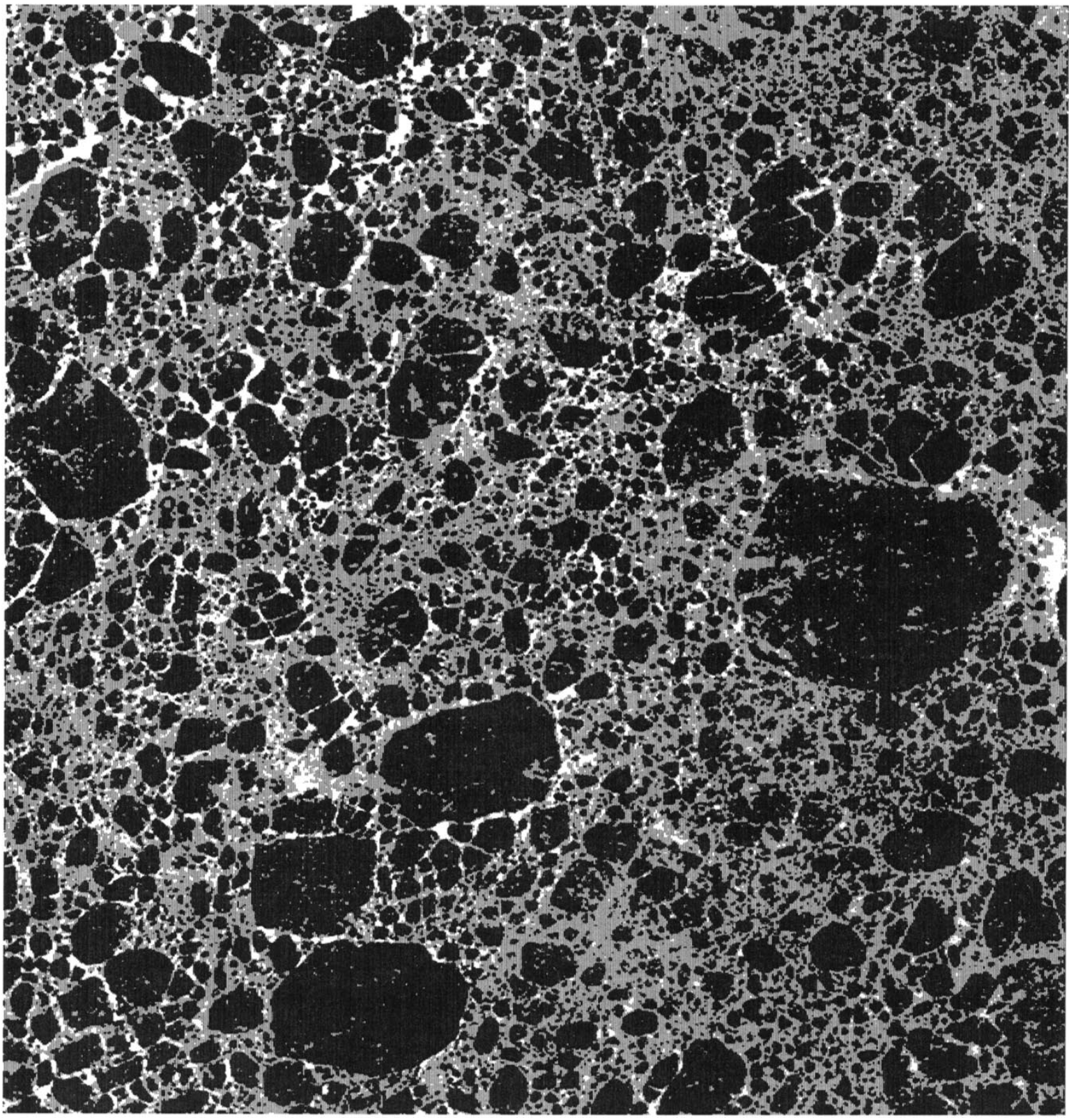

Fig. 6. Result of dynamic local thresholding of image of Fig. 5. The three different classes found are designated by black, gray, and white.

the parameterization stage indicate where floes are likely to be found. Working on only those portions of the image, a feature refinement procedure can be utilized to isolate floe shapes within the image.

The method used to distinguish floe shapes in the image is a combination of morphological techniques and a specialized object-growing method which succeeds in growing eroded objects back to their original sizes while denying them the ability to merge with nearby objects from which they may have been separated in the initial probabilistic labeling phase [21]. The purpose of this floe-refinement procedure is to improve the floe shapes in the image in three ways:

1) objects that are composed of different segment pixels become more solidly composed of object pixels;

2) objects that are composed of adjacent, disconnected parts due to lost detail during the image segmentation are grown together;
3) objects that are linked by pixels misidentified as part of objects during the processing of the image are separated into individual objects.

The result of the growing algorithm can be seen in Fig. 4 . Fig. 4(a) is the original image; Fig. 4(b) is the three-level segmented image generated for that original image; and Fig. 4(c) is the result of the floe refinement algorithm. Note the improvement of visible feature boundaries between the features in Fig. 4(c) over the boundaries in Fig. 4(b). After ice floe shapes have been refined, the image is ready for class labeling.

\section{Classification and Generation of Image Statistics}

According to the parameters supplied by the parameterization process, the three-level segmented and floe-extracted or the two-level segmented image can be labeled. It is at this point that floe shapes are also analyzed, if applicable. 


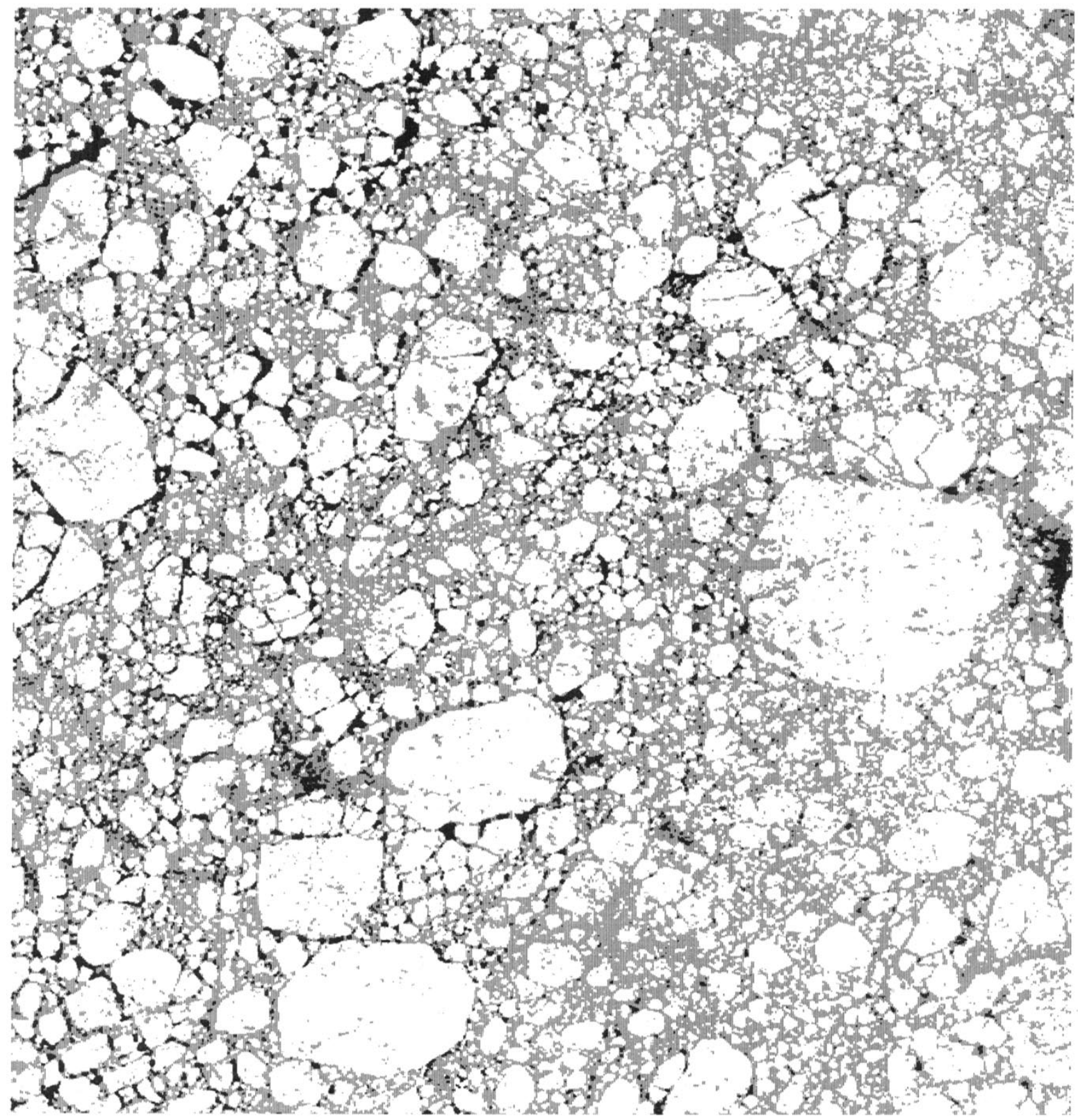

Fig. 7. Result of class labeling of the image of Fig. 6, after floe refinement and filtering. White is ice floes, gray is background matrix, and black is open water.

Floes that do not exhibit proper shape characteristics are discarded and assumed to be of the background matrix class (the backscatter of the ice matrix often overlaps with that of ice floes throughout the summer season). The nonfloe portions of the image are labeled as water and matrix or, conversely, if a two-level segmentation was performed, the image is labeled as simply water and ice.

In order to determine whether or not a floe has a proper shape, different geometric characteristics which could be extracted were experimented with to see what measure could be used to reliably filter out poorly-shaped floes (essentially, nonfloes). The calculation identified as most helpful in this task was found a measure of the branch-factor of a floe. Typically, floes are compact in shape and tending more toward round or oval than toward branchiness. A good approximation of the branch-factor of a floe can be found through a comparison of its perimeter to its area, or

$$
B_{f}=\frac{P_{f}}{4 * \sqrt{A_{f}}}
$$

which is given by [22] as a measure of compactness. According to this approximation, a square will give a branch factor of one. The higher the branch factor produced by a floe, the more branchy and less compact it is. If its branch factor exceeds some threshold, it can be considered a nonfloe and relabeled to the background matrix class.

Prior knowledge from the parameterization stage makes the relative backscatter of the resident classes immediately accessible. All features identified in the image are labeled with the appropriate class based upon a comparison between their "original" value as given by the segmentation and the class designations indicated by the parameterization stage. The segmentation gives "bright," "medium," and "dark" classes; the 
parameterization step indicates to what labels these backscatter classes correspond (floes, background matrix, and water or ice and water).

Given the labeled features and the filtered floes, we calculate ice percentages for the image. The areas of the ice floe-labeled features and the background matrix-labeled features can be added together to get the total area of the image which is covered by ice. The area of the water-labeled features can be added together to get the total area of the image which is covered by open water. These numbers are expressed as percentages of area coverage and are used in the subsequent validation step.

After faulty floe shapes have been filtered out, the floe sizes are measured and floe size distributions are generated for the image. The size bins of summer floe size distributions used are indicated in Table I. As each floe is considered, the corresponding size bin is incremented, and the total area represented by floes of that size is incremented. At the end, the area for each bin is converted into a percentage of the image area. These floe distributions can be thought of as an intermediate classification product and are used in the subsequent validation step.

\section{E. Validation}

To minimize the possibility of generating incorrect classifications, historical knowledge of the area's ice percentages and prior ice floe distributions are used as guidelines for current ice percentages and floe distributions.

1) Historical Knowledge: The National Ice Center (NIC) released a CD-ROM containing Arctic and Antarctic sea ice weekly data from 1972 through 1994 [23]. Historically, all NIC sea ice analyzes have been produced through the integration of remotely sensed and in-situ oceanographic and meteorological data. Today, sea ice analysis at the NIC is done almost exclusively with remotely sensed data. The satellites and sensors used to produce global sea ice analyzes included: TIROS visible/infrared GAC/LAC/HRPT (VHRR and AVHRR) data; NIMBUS passive microwave (ESMR and SMMR) data; DMSP visible/infrared (OLS) smooth and fine data; GEOSAT altimetry data; DMSP passive microwave (SSM/I) data; ERS-1 Synthetic Aperture Radar (SAR) data; and RADARSAT SAR data. The percent utilization of each data type varies both temporally and spatially in the weekly analysis files. For example, summer ice analyses during the period 1972-1983 were based on 60\% visible/infrared data, $30 \%$ microwave data and $10 \%$ conventional (aerial or in-situ) observations.

By analyzing this data, we generated acceptable upper and lower bounds for ice coverage for any latitude and longitude point of the Beaufort Sea for any week of the year. The variance $E\left\{x^{2}\right\}-\{E[x]\}^{2}$ for each week and location was then calculated to obtain the standard deviation of the ice concentration over the 23 years of data. An estimate for the acceptable range $R$ of ice concentrations at a given latitude La and longitude Lo for a given week $W$ of the year was expressed as:

$$
R_{W, \mathrm{La}, \mathrm{Lo}}=I_{A_{W, \mathrm{La}, \mathrm{Lo}}} \pm \sigma_{W, \mathrm{La}, \mathrm{Lo}}
$$

\begin{tabular}{|c|c|c|}
\hline \multicolumn{3}{|c|}{ Image number 25026} \\
\hline \multicolumn{3}{|c|}{ Total floes: 1823} \\
\hline Bin\# & \#floes & area_covered \\
\hline 0 & 917 & $4.89 \%$ \\
\hline 1 & 692 & $14.54 \%$ \\
\hline 2 & 203 & $25.87 \%$ \\
\hline 3 & 7 & $4.61 \%$ \\
\hline 4 & 3 & $4.861 \%$ \\
\hline 5 & 1 & $3.83 \%$ \\
\hline \multicolumn{3}{|c|}{ Image number 25026} \\
\hline \multicolumn{3}{|c|}{ Water $=5.21 \%$} \\
\hline \multicolumn{3}{|c|}{ Substratc $=36.16 \%$} \\
\hline \multicolumn{3}{|c|}{ Floc $=58.62 \%$} \\
\hline
\end{tabular}

Fig. 8. Output floe analysis and ice coverage percentages for image shown in Fig. 7.

where $I_{A}$ is the average ice concentration and $\sigma$ is the standard deviation of 23 years of sea ice concentration data. If the concentration of ice coverage resulting from the classification falls outside this range, it indicates a possible error in the classification.

2) Floe Distributions: Over the course of the summer, ice floes should be gradually melting and becoming increasingly smaller. In comparison to the most recent floe distributions measured over the same area, a current floe distribution of the area should show an overall decrease in floe sizes, or at the very least not an increase in floe sizes. If the current floe distribution does not adhere to this logic, then the user is notified and asked to supply the correct classification.

The most recent known floe size distribution for the area is taken from our database. Because the summer MIZ is a dynamic area, characterized by a high degree of ice motion, floe distributions may be used to offer only a gentle constraint: that the current floe size distribution cannot exhibit a greater coverage of larger floe sizes than the previous distribution of the same area. Using the percentage of area covered by floes of any particular size, we can compute an average floe size for a scene, using the total percentage area covered by floes to normalize. If the current average floe size is larger than the previous, then it is possible that an error has been made in the classification, since the floes should be shrinking as summer continues and ice melt progresses.

Assuming there are no errors found in the validation process, the ice database is updated with the new classification percentages and the new floe size distributions for the area. Alternatively, if errors are found, then the raw and classified images are presented to the user for either validation or correction, and the ice database is updated accordingly with the user-input classification via the database interface.

\section{System Testing AND RESUltS}

The data used for testing of the system was a time series of ERS-1 data from the Beaufort Sea, ranging from $-140^{\circ}$ to $-145^{\circ} \mathrm{E}$ longitude and from $70^{\circ}$ to $75^{\circ} \mathrm{N}$ latitude. The data are from strips imaged on four different days in August of 1991 , on Julian days 229, 232, 235, and 238. The images 


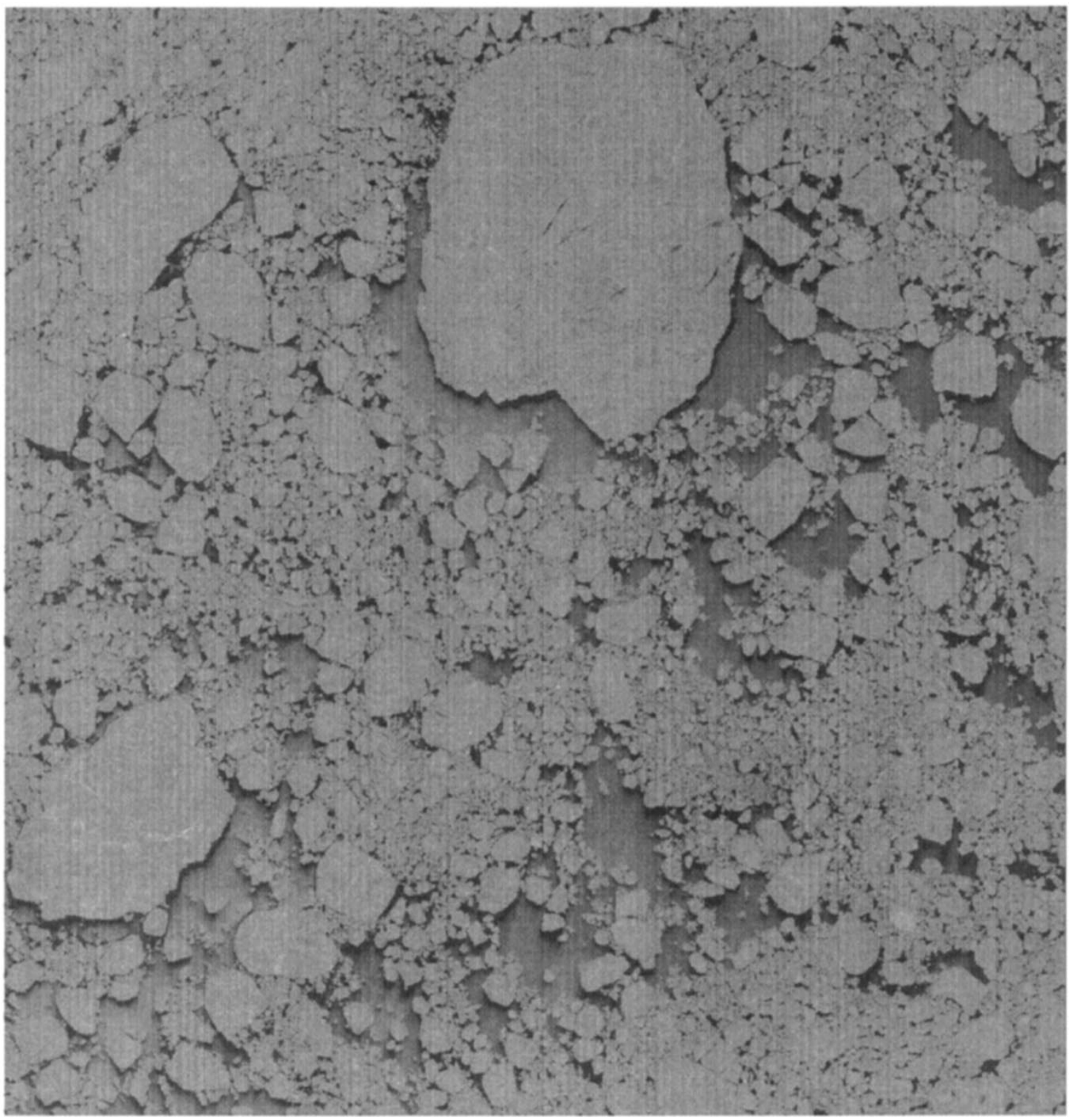

Fig. 9. Original ERS-1 image, median filtered, identification number 25363 (@ ESA 1991).

represent an area approximately $100 \times 100 \mathrm{~km}^{2}$. Of these data, 16 images were judged to be marginal ice zone imagery, appropriate for testing the system. The system was executed on the dataset in temporal order so that the classification results at a given latitude and longitude point could be used to assist in the classification of the temporally successive image at that same location.

\section{A. Evaluation Methods}

The classification of each image in the set was evaluated on a scale of $0-4$ on the quality of the visual classification. Factors that were taken into account were as follows, listed in order of importance.

1) Correctness of assigned classes. Was the coverage type (floes, matrix, water or ice, water) assigned to the proper segmented class? The focus of the work is achieving a correct, automated classification.

2) Correctness of segmentation. Are the ice and water classes consistent with the original image? Distinct floe, matrix, and water areas should agree with the original image. When the floes and matrix mix, the ice (ice floes and background matrix) and water should be separate.

3) Quality of extracted floes (if any). Are floe shapes visually apparent in the original image present in the classified image? Floe statistics are generated for use in later classification of the same area.

4) Rejection of floes with nonfloe shapes. Are floes with nonfloe shapes rejected by the floe analysis and classification procedure? Poor floe shapes will affect the floe statistics adversely. 


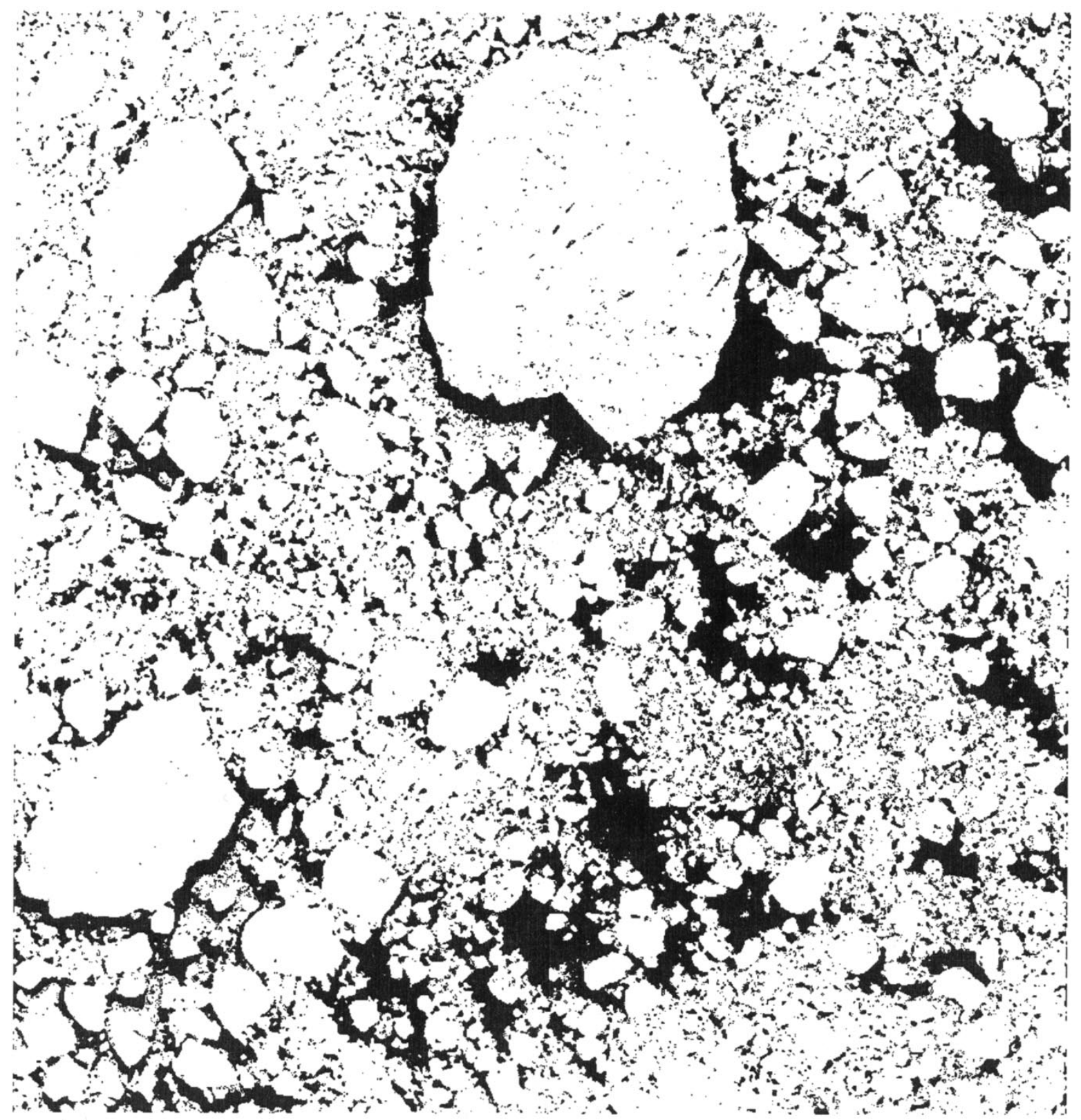

Fig. 10. Result of neighbor-based segmentation followed by class labeling of the image of Fig. 9. White is ice (ice floes and background matrix together) and black is open water.

The evaluation of each image and the total average scores are shown in Table II. The evaluations of two separate judges were averaged to score each image.

\section{B. Discussion of Results}

Generally speaking, the results of the system were very good. The fusion of various types of information with the imagery, and the inclusion of the information at appropriate stages of the image analysis process, proved to correctly guide the algorithm. The parameterization stage produced the relative expected backscatters that allowed the automated classification of the image. In cases where a three-level segmentation and classification was considered feasible, the dynamic local thresholding technique preserved contrast in the image, enabling good floe separation. This was followed by a good improvement of floe shapes through the floe refinement technique, and a final classification that was consistent with visual image inspection. In cases where only separation in ice and water was feasible, the neighbor-based segmentation provided good distinction between ice and water areas and a final classification that was also consistent with visual image inspection. To provide examples of the results of the automated classification procedure, two of these images were selected for presentation and discussion.

Day 229: Image \#25 026

This scene, centered at $74^{\circ} \mathrm{N}, 143^{\circ} \mathrm{W}$, was under advanced melt (information established from the temperature database) with a wind speed of more than $5 \mathrm{~m} / \mathrm{s}$. The original image can be seen in Fig. 5. Notice the bright water, indicating a high wind speed. Notice also the variation in the backscatter of the 


\section{Image number 25363 \\ Total flocs: ? \\ Image number 25363 \\ Water $=23.20 \%$ \\ $\mathrm{Icc}=76.80 \%$}

Fig. 11. Output analysis of ice coverage for image shown in Fig. 9. Since the two-level segmentation was used, no floe statistics were computed.

water across the image. Upon execution, the parameterization stage resulted in invocation of a three-level segmentation and classification of the image, assuming dark floes, medium background matrix, and bright water. The subsequent dynamic local thresholding produced the image of Fig. 6. Comparing Fig. 6 to Fig. 5 one notices the preservation of detail achieved by the local dynamic thresholding technique. After a subsequent floe refinement and class labeling, the three-level classified image is as depicted in Fig. 7. The ice coverage of $94.78 \%$ survived the comparison to historical data, and because there were no prior floe statistics for the area, the floe distribution was accepted as correct. Statistics and ice percentages generated for the image can be seen in Fig. 8. The three classes (floes, matrix, and water) are assigned properly; the segmentation itself resulted in good separation among classes: the ice regions and water regions were divided properly and good floe shapes were preserved.

Day 238: Image \#25363

This scene, centered at $74^{\circ} \mathrm{N}, 144^{\circ} \mathrm{W}$, was under advanced melt with a wind speed of less than $5 \mathrm{~m} / \mathrm{s}$. The original image is shown in Fig. 9. Notice the wide backscatter range of the water in the image, from dark to bright portions. Upon execution, the information generated by the parameterization stage guided the system to select the two-level segmentation and classification algorithm. The subsequent neighbor-based segmentation and subsequent class labeling produced the image of Fig. 10. Compared to Fig. 9, Fig. 10 shows good separation between ice and water areas. The ice coverage of $76.80 \%$ failed the comparison to historical information, which stated the acceptable range as $86.20 \%$ to $100.00 \%$. Because no floe sizes could be generated for this image, no comparison to prior floe sizes was performed. Statistics and ice percentages generated for the image are shown in Fig. 11. In this case, the user was notified to evaluate the results of the algorithm and she accepted the system's output as correct.

\section{CONCLUSION}

The approach to the problem of classifying data with temporally inconsistent backscatter involves the fusion of a number of information sources in addition to the intensity data itself in order to attain an automated classification. Different types of ancillary data were identified and interpreted into a usable format to help define a context for SAR ERS-1 data that allowed its segmentation and automated labeling. The method for doing this involves time analyses of certain data sources, the conversion of those analyses into information, and the use of this information in guiding a number of different algorithms through the segmentation and classification of a given image. Statistical historical information and temporal data (recent prior classification results) are used to validate this classification before accepting it as correct. In the event that the assumed classification fails the validation step, the user is allowed to input correct classification values. The possibility of user intervention is beneficial in that it helps prevent the system from being corrupted with incorrect feedback data.

The information fusion approach was used to analyze SAR images of the summer MIZ. We combined information fusion with good segmentation techniques and validation checks based upon past historical information and expected floe behavior, and our test results showed that the system generated very good results.

The historical ice concentration database is currently limited to a section of the Beaufort Sea. To realize the full potential of this system, historical information for latitude and longitude points in all regions of the Arctic Seas should be analyzed and incorporated into the historical database. Currently, the area of application is small, and the system is not suited for large-scale use. Results on this subset of the Arctic Oceans, however, show a positive potential for the use of information fusion in the interpretation of remotely sensed data.

\section{REFERENCES}

[1] G. A. Maykut, "Energy exchange over young sea ice in the central arctic," J. Geophys. Res., vol. 88, pp. 3646-3658, 1978.

[2] S. D. Smith, R. D. Muench, and C. H. Pease, "Polynyas and leads: An overview of physical processes and environment," J. Geophys. Res., vol. 95, pp. 9461-9479, 1990.

[3] D. P. Winebrenner, D. G. Long, and B. Holt, "Mapping the progression of melt onset and freeze-up on arctic sea ice using SAR and scatterometry," in Analysis of SAR Data of the Polar Oceans, C. Tsatsoulis and R. Kwok, Eds. New York: Springer-Verlag, 1998, pp. 129-144.

[4] C. E. Livingstone, K. P. Singh, and A. L. Gray, "Seasonal and regional variations of active/passive microwave signatures of sea ice," IEEE Trans. Geosci. Remote Sensing, vol. GE-25, pp. 159-172, Mar. 1987.

[5] D. G. Barber, A. Thomas, and T. N. Papakyriakou, "Role of SAR in surface energy flux measurements over sea ice," in Analysis of SAR Data of the Polar Oceans, C. Tsatsoulis and R. Kwok, Eds. New York: Springer-Verlag, 1998, pp. 35-67.

[6] R. G. Onstott, T. C. Grenfell, C. Matzler, C. A. Luthre, and E. A. Svendsen, "Evolution of microwave sea ice signatures during early summer and midsummer in the marginal ice zone," J. Geophys. Res., vol. 92, no. C7, pp. 6825-6835, June 1987.

[7] E. Waltz and J. Llinas, Multisensor Data Fusion. Boston, MA: Artech House, 1990.

[8] P. D. Heermann and N. Khazenie, "Classification of multispectral remote sensing data using a back-propagation neural network," IEEE Trans. Geosci. Remote Sensing, vol. 30, pp. 81-88, Jan. 1992.

[9] J. D. Paola and R. A. Schowengerdt, "A detailed comparison of backpropagation neural network and maximum-likelihood classifiers for urban land use classification," IEEE Trans. Geosci. Remote Sensing, vol. 33, pp. 981-996, July 1995.

[10] J. A. Benediktsson and P. H. Swain, "Methods for multisource data analysis in remote sensing," Tech. Rep. TR-EE 87-26, Lab. Applicat. Remote Sensing, Sch. Elect. Eng., Purdue Univ., West Lafayette, IN, 1987.

[11] E. Rignot, R. Chellappa, and P. Dubois, "Unsupervised segmentation of polarimetric SAR data using the covariance matrix," IEEE Trans. Geosci. Remote Sensing, vol. 30, pp. 697-705, July 1992.

[12] J. S. Miguel-Ayanz and G. S. Biging, "An iterative classification approach for mapping natural resources from satellite imagery," Int. J. Remote Sensing, vol. 17, no. 5, pp. 957-981, 1996.

[13] V. Clement, G. Giraudon, S. Houzelle, and F. Sandakly, "Interpretation of remotely sensed images in a context of multisensor fusion using a 
multispecialist architecture," IEEE Trans. Geosci. Remote Sensing, vol. 31, pp. 779-791, July 1993.

[14] S. G. Beaven and S. P. Gogineni, "Fusion of satellite SAR with passive microwave data for sea ice remote sensing," in Analysis of SAR Data of the Polar Oceans, C. Tsatsoulis and R. Kwok, Eds. New York: Springer-Verlag, 1998, pp. 91-109.

[15] S. P. Gogineni, R. K. Moore, T. C. Grenfell, D. G. Barber, S. Digby, and M. Drinkwater, "The effects of freeze-up and melt processes on microwave signatures," in Geophysical Monograph 68: Microwave Remote Sensing of Sea Ice, F. D. Carsey, Ed. Washington, DC: American Geophysical Union, pp. 329-341, 1992.

[16] R. G. Onstott and S. P. Gogineni, "Active microwave measurements of arctic sea ice under summer conditions," J. Geophys. Res., vol. 90, no. C3, pp. 5035-5044, May 1985.

[17] D. P. Winebrenner, D. G. Long, and B. Holt, "Mapping the progression of melt onset and freeze-up on arctic sea ice using SAR and scatterometry," in Analysis of SAR Data of the Polar Oceans, C. Tsatsoulis and R. Kwok, Eds. New York: Springer-Verlag, 1998, pp. 129-144.

[18] Holt, B. G. Cunningham, and R. Kwok, "Sea ice radar signatures from ERS-1 SAR during late summer and fall in the Beaufort and Chukchi seas," presented at First ERS-1 Symp., Cannes, Nov. 4-6, 1992.

[19] C. K. Chow and T. Kaneko, "Automatic boundary detection of the left ventricle from cineangiograms," Comput. Biomed. Res., vol. 5, pp. 388-410, 1972.

[20] D. Haverkamp, L. K. Soh, and C. Tsatsoulis, "A dynamic local thresholding technique for sea ice classification," in Proc. Int. Geoscience Remote Sensing Symp., 1993, pp. 638-640.

[21] L. K. Soh, B. Holt, and C. Tsatsoulis, "Ice floe separation: The restricted growing concept and its application in floe size distribution for SAR sea ice imagery," in Analysis of SAR Data of the Polar Oceans, C. Tsatsoulis and R. Kwok, Eds. New York: Springer-Verlag, 1998, p. 34.

[22] A. K. Jain, Fundamentals of Digital Image Processing, Englewood Cliffs, NJ: Prentice-Hall, 1989, pp. 393-394.
[23] National Ice Center, Fleet Numerical Meteorology and Oceanography Detachment, and National Climatic Data Center, Arctic and Antarctic Sea Ice Data 1972-1994, CD-ROM Version 1.0, July 1996.

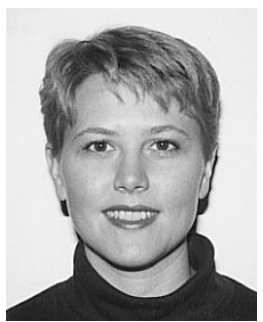

Donna Haverkamp (S'91-M'92) received the $\mathrm{Ph} . \mathrm{D}$. degree in electrical engineering from The University of Kansas, Lawrence.

She is currently with the Information Systems Division, Harris Corporation, Melbourne, FL. Her research interests are in image processing, computer vision, and artificial intelligence. She is particularly interested in applying artificial intelligence to problems traditionally dealt with in the field of image processing.

Kappa $\mathrm{Nu}, \mathrm{ACM}$, and AAAI.

Dr. Haverkamp is a member of Tau Beta Pi, Eta

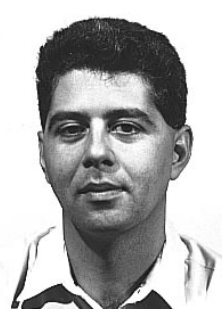

Costas Tsatsoulis (M'88-SM'98) received the $\mathrm{Ph} . D$. degree in electrical engineering in 1987 from Purdue University, West Lafayette, IN.

$\mathrm{He}$ is currently an Associate Professor, Department of Electrical Engineering and Computer Science, The University of Kansas, Lawrence. His research interests include satellite image processing and interpretation, data mining, and multiagent systems. He recently co-edited (with R. Kwok) the book Analysis of SAR Data of the Polar Oceans (New York: Springer-Verlag, 1998).

Dr. Tsatsoulis is a member of ACM, AAAI, Eta Kappa Nu, and Sigma Xi. 